\title{
Morphological and Chemical Composition Characterization of Commercial Sepia Melanin
}

\author{
Agnes Mbonyiryivuze ${ }^{1,2, *}$, Z. Y. Nuru ${ }^{1,2}$, Balla Diop Ngom ${ }^{1,3}$, Bonex Mwakikunga ${ }^{1,4}$, Simon Mokhotjwa Dhlamini ${ }^{1,5}$, \\ Eugene Park $^{1,2,6}$, Malik Maaza ${ }^{1,2}$ \\ ${ }^{1}$ UNESCO-UNISA Africa Chair in Nanosciences/Nanotechnology, College of Graduate Studies, \\ University of South Africa, Pretoria-South Africa \\ ${ }^{2}$ Nanosciences African Network (NANOAFNET), iThemba LABS-National Research Foundation, Cape Town, South Africa \\ ${ }^{3}$ Laboratoire de Photonique et de nanofablication, Groupe de Physique du Solide et des Science matériaux (GPSSM), Faculté de \\ Science et Techniques, Université Cheikh Anta Diop de Dakar(UCAD), Dakar, Sénégal \\ ${ }^{4}$ CSIR- National Centre for Nano-Structured Materials, Pretoria, South Africa \\ ${ }^{5}$ Department of Physics, Florida Research Centre, University of South Africa, Florida-South Africa \\ ${ }^{6}$ Materials Sci. \& Eng. Department, Nelson Mandela African Institute of Science \& Technology, Arusha-Tanzania \\ *Corresponding author: mbonyiryivuzeagnes@yahoo.com
}

Received May 08, 2015; Revised May 22, 2015; Accepted June 25, 2015

\begin{abstract}
Melanins are difficult to characterize because of their intractable chemical properties and the heterogeneity in their structural features. Melanin pigments, in fact, are composed of many different types of monomeric units that are connected through strong carbon-carbon bonds. Its high insolubility and undefined chemical entities are two obstacles in its complete characterization. The morphological characterization and particle size distribution for sepia melanin by Scanning Electron Microscopy (SEM) on surface structure and Transmission Electron Microscopy (TEM) to confirm the morphology obtained from SEM was done. Both results show that Sepia melanin is formed by many aggregates agglomerated together. These aggregates are formed also by small spherical granules with different size distributions that have been determined using image-J software. The small granule diameter obtained from different TEM and SEM micrographs were 100-200nm. EDS reveals that C and O were the most abundant in sepia melanin with concentration average concentrations of about $57 \%$ and $24 \%$ respectively. The major compositions of sepia melanin are $\mathrm{C}, \mathrm{O}, \mathrm{Na}, \mathrm{Cl}$, while the minor are $\mathrm{Mg}, \mathrm{Ca}, \mathrm{K}, \mathrm{S}$ and $\mathrm{N}$. From TEM micrograph at high resolution, it was possible to measure the distance between polymers layers of sepia melanin using image-J software and it was $0.323 \mathrm{~nm}=3.23 \mathrm{~A}$.
\end{abstract}

Keywords: melanin, sepia melanin, eumelanin, SEM, TEM, sepia officinalis

Cite This Article: Agnes Mbonyiryivuze, Z. Y. Nuru, Balla Diop Ngom, Bonex Mwakikunga, Simon Mokhotjwa Dhlamini, Eugene Park, and Malik Maaza, "Morphological and Chemical Composition Characterization of Commercial Sepia Melanin.” American Journal of Nanomaterials, vol. 3, no. 1 (2015): 22-27. doi: 10.12691/ajn-3-1-3.

\section{Introduction}

Melanins are biopolymers widely distributed in many parts of living organisms. They constitute a major group of biological polymers responsible for much of diversity of coloration in animal world. Melanins are usually classified in eumelanins and pheomelanin. Eumelanin are dark pigments containing nitrogen while pheomelanins are yellow to reddish-brown containing sulfur $[1,2,3]$. Eumelanin and pheomelanin are both found in the skin, hair and eyes of many animal species, including humans, where they act as photoprotectants (absorbing harmful ultraviolet and visible radiation). Dark-skinned people have more melanin in their skin than light-skinned people have $[4,5]$.

Melanins have been reported to have a diverse number of functions in the biosystem, including photosensitization, metal ion chelation, photoprotection to absorb a broad range of electromagnetic radiation, antibiotic, thermoregulation [6]. Melanins are found all over the body from the skin and blood to the nervous system but the role of melanin in all these system is unclear. However, some studies come out with some of its biological functions among them the main one was photoprotection $[7,8]$. Melanin has important role in the skin's barrier function by preventing damage by UV radiation. It absorbs UV radiation, thus protecting the cell's nuclei from DNA (Deoxyribonucleic acid) damage $[9,10]$.

Melanins also have applications in agriculture, medicine, cosmetic and pharmaceutical industries [8]. Many commercial products contain melanin as active ingredients; including creams that act as filters for singleresponse protection against UV radiation [11]. Melanins are used in cosmetics to fade defects of the skin diseases called 'vitiligo' which is caused by a loss of melanin in the skin, due to destruction of melanin-forming cells 
known as melanocytes [5,12]. The production of sunglasses with a high ability to block UV radiations has been done by adding melanin to plastics. Melanin are also used to prevent damage to objects in museums or libraries because when coated to the internal surface of fluorescent lamp, they eliminate entirely the escape of UV light which usually occurs at a low level in these lamps [13]. The melanin is also consumed in enjoying food from squid in its ink [2]. Due to the presence of melanin polymer in sepia ink, sepia ink possesses antimicrobial activity [14]. Melanin coating is model of coating for both biological investigations and smart surface science [15].

Sepia melanin is made of eumelanin refer the melanin isolated from ink sac of the cuttlefish Sepia officinalis and it has been proposed as a standard for natural melanin [1], [14]. Sepia melanin is insoluble in organic solvents, acids, aqueous solutions, and only partially dissolves in alkaline solutions. Sepia ink is the ink extracted from Sepia officinalis. This ink contains $\mathrm{CaCO}_{3}, \mathrm{MgCO}_{3}, \mathrm{NaCl}$ and $\mathrm{Na}_{2} \mathrm{SO}_{4}$, enzymes and other substances $[13,14]$. Purified sepia melanin is a black powder and so hygroscopic that should be refrigerated at $-20^{\circ} \mathrm{C}$ to avoid decomposition.

For other essential biopolymers such as proteins, nucleic acids as well as carbohydrates, they are well characterized. Their monomeric units and connectivity are well known and the methodologies for determination of the sequences of their connection are well-established. In contrary, structures of melanin are still unknown because till now; no available methods allowing to accurately determine their structures [7,11,12].

There are many factors hindering accurate characterization of melanin and some are the following: Melanins are insoluble in a broad range of solvents and $\mathrm{pH}$. Their purification is difficult which leads to the heterogeneity in their structural features. In addition, the methods to accurately determine the ratio of the various units present in melanin are not yet found. The molecular structure and organization of melanin are complicated and not completely known [7,16]. A great number of investigations on melanin have been done, but till now it is still not well understood. An accurate definition of melanin biopolymer does not exist as its structure is not well known till now [17,18,19].

It is expected that there will be many new future applications and products that are based on melanins and this will lead to the increase in demand for melanin. There are many researches being carried out on melanin. Fortunately, melanin is abundant because it can be extracted from animal tissues and plants at low cost [2]. One of the challenges for this method is that the melanin obtained generally has low purity and there is variation in composition in each batch. However, this melanin can also be synthesized by chemical methods which guarantee its purity.

Melanin is of interest as a model system of understanding disorder in biological systems. The biological functionality of melanin depends on disorder which is considered as its essential part. This property distinguishes melanin from other much more intensively studied biomolecule systems such as nucleic acid, proteins and carbohydrates [20]. This paper report the results on morphological structure of sepia melanin obtained from SEM and TEM as well as its elemental composition obtained from EDS.

\section{Materials and methods}

\subsection{Materials}

Sepia melanin powder (standard) from Sepia officinalis was obtained from Sigma-Aldrich (Chemie $\mathrm{GmbH}$ Kappelweg 1 D-91625 Schnelldorf, Germany). This purified sepia melanin is a black powder, hygroscopic that has been kept refrigerated at $-20^{\circ} \mathrm{C}$ to avoid any photochemical of photo-physical alterations. Acetone (99\%) was used Transmission Electron Microscopy (TEM), it was used to make sepia melanin suspension.

\subsection{Characterisation techniques}

The morphological characterization and particle size distribution for sepia melanin has been done by Scanning Electron Microscopy (SEM) on surface structure and Transmission Electron Microscopy (TEM) to confirm the morphology obtained from SEM. Chemical composition of sepia melanin has been done by EDS (Energy dispersive X-ray Spectroscopy).

The sepia melanin powder was coated with goldpalladium (Au: Pd; 6O: 40) layer using a coating sputter coater (Quorum Q 150 TES) to increase the conductivity of the sample. SEM and EDS were performed on same sample using Carl Zeiss Auriga Field Emission Scanning Electron Microscope (FEG SEM) imaging at $5 \mathrm{keV}$. EDS spectra was collected with an OXFORD instruments XMAX solid state silicon drift detector at $20 \mathrm{keV}$.

For further investigation on the morphology of sepia melanin in order to confirm the results obtained by SEM, the Fei Tecnai $G^{2} 20$, TEM having Field Gun Resolution TEM operating at $200 \mathrm{kV}$ with an information limit of $0.12 \mathrm{~nm}$ was used. Sample for TEM analysis were prepared by depositing a small drop of dilute dispersion of sepia melanin ultrasonicated in acetone TEM grids. The TEM grids were coated with a thin amorphous carbon support ( 20nm); as it has a relatively low electron density, it provides a uniform substrate for imaging samples. The sample preparation procedure used was the one used by of Watt et al. except that the solvent was acetone as solvent.

For both SEM and TEM characterizations, the size particle distribution was measured using particle size analysis software called image-J. The image-J software can calculate the area and pixel value statistics of userdefined selection and intensity threshold objects. It can also measure the spacing between the lines as well as the length from TEM and SEM micrographs. While SEM micrographs display compositional contrast that results from different atomic number elements and their distribution, EDS allows the identification of what those particular elements are and their relative proportions.

EDS analysis usually involves the generation of an Xray spectrum from the entire scan area of the SEM [21]. From EDS, we get the corresponding X-ray spectra that were generated from the entire scan area by SEM. The Yaxis shows the counts (number of $\mathrm{X}$-rays received and processed by the detector) and the $\mathrm{X}$-axis shows the energy level of those counts [21,22]. The EDS qualitative analysis consists of the identification of the lines in the $\mathrm{x}$ ray spectrum using tables of energies or wavelengths and the EDS quantitative analysis (determination of the 
concentrations of the elements present) by measuring line intensities for each element in the sample and for the same elements in calibration standards of known composition [23]. The X-ray data booklet was used to analyse EDS results on sepia melanin.

\section{Results and Discussion}

\subsection{SEM Micrographs}

At a scale of $10 \mu \mathrm{m}$ and $1.00 \mathrm{~K} \mathrm{X}$ magnification, sepia melanin appeared to be formed by aggregates with different sizes that are grouped together (Figure 1a). In increasing the magnification 10 times and reducing the scale to $1 \mu \mathrm{m}$, the aggregates seem to be formed by small granules (Figure 1b). When the magnification is increased further up to $20.00 \mathrm{~K} \mathrm{X}$ at the same scale, the small granules forming the aggregates start to be a little beat clear (Figure 1c). Due to the increasing of magnification further up to $50 \mathrm{~K} \mathrm{X}$, and change the scale from micrometers to nanometers order (from $1 \mu \mathrm{m}$ to $200 \mathrm{~nm}$ ), granules start appearing to be spherical (Figure 1d).
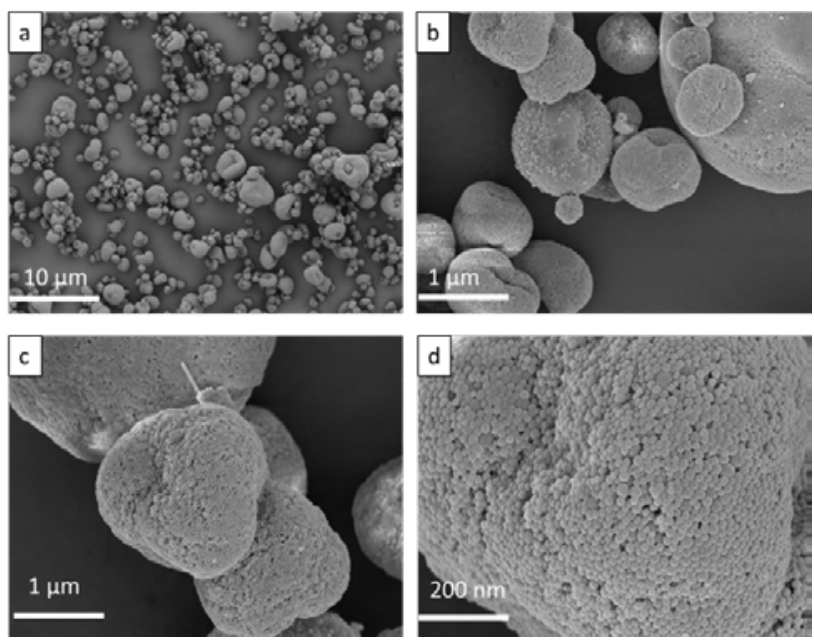

Figure 1. SEM micrographs of sepia melanin granules at $1.00 \mathrm{~K} \mathrm{X}$ (a), 10.00K X (b), 20.00 K X (c) and 50.00 K X (d) magnifications.

The low resolution SEM micrographs of the sepia melanin sample are shown on Figure 1. Sepia melanin is formed by many aggregates agglomerated together. These aggregates are formed also by small spherical granules with different size distributions and this start to become clear at the higher resolution.

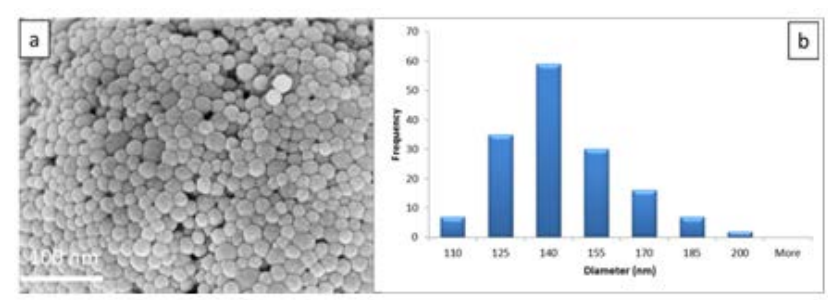

Figure 2. SEM micrographs of sepia melanin granules at $100.00 \mathrm{~K} \mathrm{X}$ magnification

The diameter distribution measured from SEM micrograph (Figure 2a) is represented on the histogram (Figure 2b).

The above histogram (Figure 3b) shows the diameter distribution of sepia melanin granules measured from
SEM micrograph (Figure 3a). It was found that sepia melanin is made of aggregate of spherical particles having about 100 - $200 \mathrm{~nm}$ in diameter as shown by the histogram (Figure 2.b and Figure 3b).

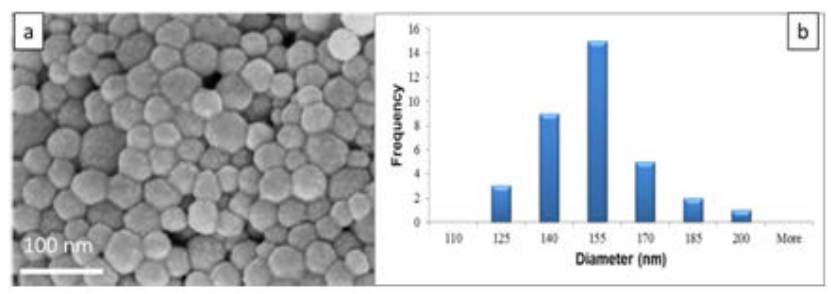

Figure 3. SEM micrographs of sepia melanin granules at $200.00 \mathrm{~K} \mathrm{X}$ magnification and the corresponding histogram of diameter distribution

Particle diameter was in the range of 100 and $200 \mathrm{~nm}$, and the calculated average diameter is $137 \mathrm{~nm}$. Kim et al. reported the average diameter of melanin nanoparticles to be $135 \mathrm{~nm}$ [6].

The obtained diameter distributions for sepia melanin from SEM micrographs were in good agreement with the ones reported in literature. It was reported that the size diameter of spherical particles forming natural melanin varies in the range of $100-200 \mathrm{~nm}$ [21,22,23]. Watt et al. as well as Bothma found that the diameters of sepia melanin granules which are roughly spherical were 100 $\mathrm{nm}$ upwards [24,25]. The diameter of the aggregated spherical granules constituting sepia melanin was reported to be about $150 \mathrm{~nm}$ [26].

\subsection{EDS Spectra}

The EDS measurement was done on five different spots from SEM micrographs (at $100 \mathrm{~K} \mathrm{X}$ magnification for all of them) to make sure that the chemical composition is the same for different positions of the sample.
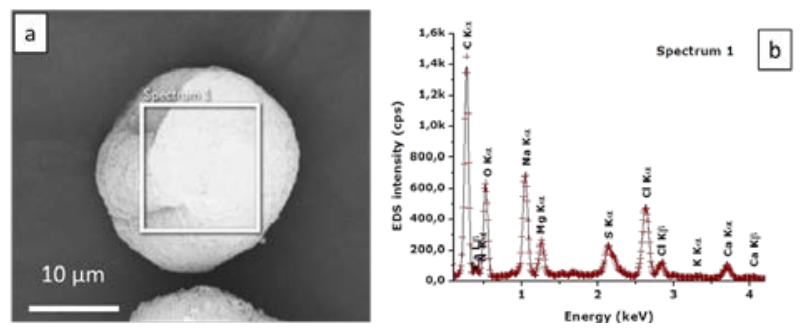

Figure 4: SEM micrographs of sepia melanin granules at $100.00 \mathrm{~K} \mathrm{X}$ magnification and $10 \mu \mathrm{m}$ scaled and the corresponding EDS spectrum

All presented EDS spectra in paper were plotted from raw data collected from EDS. The first EDS spectrum collected from SEM micrograph (Figure 4a) of sepia melanin is shown on Figure 4b. This spectrum was collected on SEM micrograph scaled at $10 \mu \mathrm{m}$ while the remaining spectra were collected from $1 \mu \mathrm{m}$ SEM micrographs scaled. On this EDS spectrum, one can distinguish the peaks of $\mathrm{C}, \mathrm{O}, \mathrm{Na}, \mathrm{Mg}, \mathrm{Cl}, \mathrm{S}, \mathrm{Ca}, \mathrm{K}$ as well as $\mathrm{N}$ even if the two last elements are low in intensity. This spectrum is the only one showing the existence of $\mathrm{K}$ element in sepia melanin among all the five spectra as the measurement was done on a big portion of the sample. The potassium was not present in all other four spectra (Figure 5, Figure 6, Figure 7, and Figure 8), while nitrogen was not only present on two of them (Figure 5 and Figure 8). 

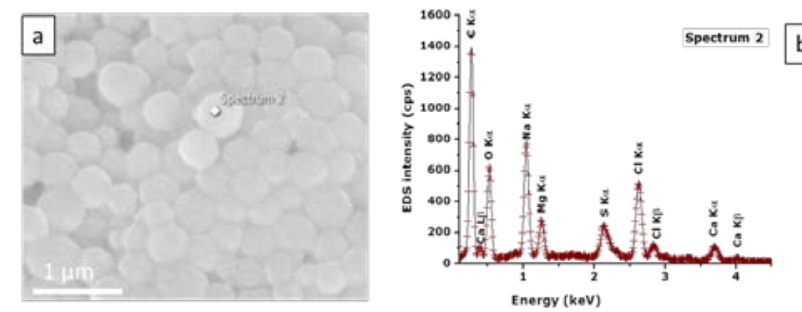

Figure 5. SEM micrographs of sepia melanin granules at $100.00 \mathrm{~K} \mathrm{X}$ magnification and the corresponding EDS spectrum

Figure $5 \mathrm{~b}$ is the EDS spectrum collected from the second spot (Figure 5a). On this spot, the detected elements were only $\mathrm{C}, \mathrm{O}, \mathrm{Na}, \mathrm{Mg}, \mathrm{Cl}, \mathrm{S}$, and $\mathrm{Ca}$. The concentrations as well as the maximum intensities of emitted $\mathrm{x}$-ray lines were different from those obtained at the first spot.
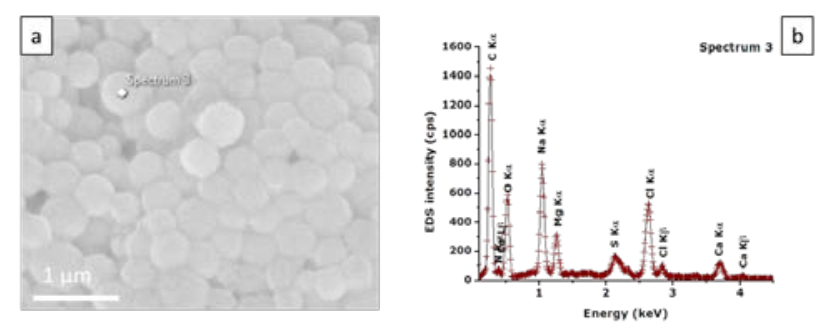

Figure 6. SEM micrographs of sepia melanin granules at $100.00 \mathrm{~K} \mathrm{X}$ magnification and the corresponding EDS spectrum

Figure 6b: EDS spectrum collected from the third spot (Figure 6a). On this spot, the detected elements were only $\mathrm{C}, \mathrm{O}, \mathrm{Na}, \mathrm{N}, \mathrm{Mg}, \mathrm{Cl}, \mathrm{S}$, and $\mathrm{Ca}$. The concentrations as well as the maximum intensities of emitted $\mathrm{x}$-ray lines were different from those obtained on other spots.
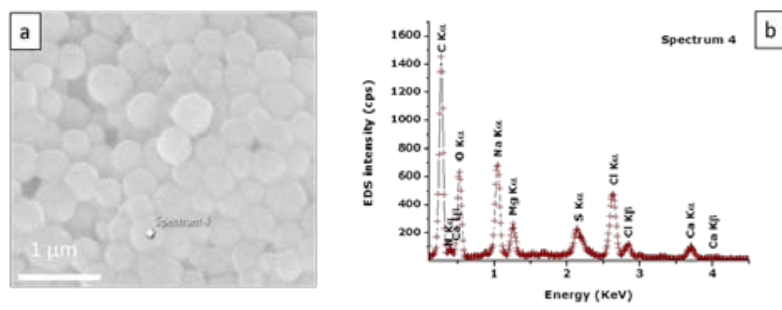

Figure 7. SEM micrographs of sepia melanin granules at $100.00 \mathrm{~K} \mathrm{X}$ magnification and the corresponding EDS spectrum

The EDS spectrum (Figure 7b) collected from the fourth spot (Figure 7a). On this spot, the detected elements were only $\mathrm{C}, \mathrm{N}, \mathrm{O}, \mathrm{Na}, \mathrm{Mg}, \mathrm{Cl}, \mathrm{S}$, and $\mathrm{Ca}$. The concentrations as well as the maximum intensities of emitted $\mathrm{x}$-ray lines were different from those obtained on other spots.
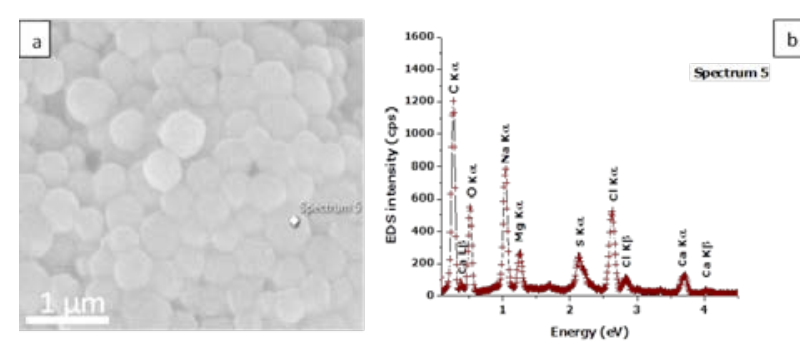

Figure 8. SEM micrographs of sepia melanin granules at $100.00 \mathrm{~K} \mathrm{X}$ magnification and the corresponding EDS spectrum
Figure $8 \mathrm{~b}$ is the EDS spectrum collected from the fifth spot (Figure 8a). On this spot, the detected elements were only $\mathrm{C}, \mathrm{O}, \mathrm{Na}, \mathrm{Mg}, \mathrm{Cl}, \mathrm{S}$, and $\mathrm{Ca}$. The concentrations as well as the maximum intensities of emitted $\mathrm{x}$-ray lines were different from those obtained on other spots.

The table below (Table 1) represent the summary of all elements detected from the five spectra, and their corresponding photon energies of $\mathrm{x}$-ray emission lines in electron volt. For each spectrum, the element and their collected maximum intensities for K-, and L-shell emission lines are presented. The results shows that the intensity of emitted x-ray lines from all five spot were not the same and this justified the difference in concentrations for all detected elements. Briefly, the detected elements are not distributed uniformly in the samples. The difference in concentration is shown on Table 2.

Table 1. Summary of all Detected Elements from the Five Spectra and their Corresponding Photon Energies of X-ray Emission Lines in Electron Volts

\begin{tabular}{|c|c|c|c|c|c|c|}
\hline Element & $\mathrm{E}$ & $\mathrm{I}_{\mathrm{S} 1}$ & $\mathrm{I}_{\mathrm{S} 2}$ & $\mathrm{I}_{\mathrm{S} 3}$ & $\mathrm{I}_{\mathrm{S} 4}$ & $\mathrm{I}_{\mathrm{S} 5}$ \\
\hline $\mathrm{C} \mathrm{K \alpha}$ & 0.277 & 1453.0 & 1471.0 & 1455.0 & 1256.0 & 1203.0 \\
\hline N K $\alpha$ & 0.392 & 90.0 & - & 79.0 & 71.0 & - \\
\hline $\mathrm{O} \mathrm{K} \alpha$ & 0.520 & 632.0 & 620.0 & 590.0 & 530.0 & 548 \\
\hline $\mathrm{Na} \mathrm{K} \alpha$ & 1.041 & 684.0 & 772.0 & 800.0 & 725.0 & 782.0 \\
\hline $\mathrm{Mg} \mathrm{K} \alpha$ & 1.254 & 265.0 & 279.0 & 800.0 & 274.0 & 260.0 \\
\hline $\mathrm{S} K \alpha$ & 2.307 & 229.0 & 247.0 & 166.0 & 153.0 & 240.0 \\
\hline $\mathrm{Cl} \mathrm{K \alpha}$ & 2.622 & 476.0 & 516.0 & 526.0 & 476.0 & 524.0 \\
\hline $\mathrm{Cl} \mathrm{K} \beta$ & 2.816 & 122.0 & 122.0 & 103.0 & 103.0 & 111.0 \\
\hline $\mathrm{K} \mathrm{K \alpha}$ & 3.314 & 36.0 & - & - & - & - \\
\hline Ca K $\alpha$ & 3.692 & 105.0 & 105.0 & 120.0 & 123.0 & 111.0 \\
\hline Сa K $\beta$ & 4.012 & 24.0 & 37.0 & 24.0 & 31.0 & 36.0 \\
\hline Ca L $\beta$ & 0.341 & 65.0 & 65.0 & 79.0 & 53.0 & 84.0 \\
\hline
\end{tabular}

On Table 1, E stands for the photon energy from x-ray data booklet while $I_{s}$ stands for the maximum intensities of the emitted $x$-ray lines peaks).The table shows how the intensities of emitted $\mathrm{x}$-ray lines differ from on spot to another.

Table 2. Concentrations of Different Elements Composing Commercial Sepia Melanin

\begin{tabular}{|c|c|c|c|c|c|c|}
\hline Element & S1 & S2 & S3 & S4 & S5 & Mean values \\
\hline $\mathrm{C}$ & 55.49 & 60.21 & 55.23 & 52.98 & 59.46 & 56.67 \\
\hline $\mathrm{N}$ & 7.14 & - & 7.06 & 8.97 & - & 4.63 \\
\hline $\mathrm{O}$ & 24.33 & 24.7 & 23.34 & 23.19 & 23.73 & 23.86 \\
\hline $\mathrm{Na}$ & 6.19 & 7.24 & 6.81 & 7.04 & 7.93 & 7.04 \\
\hline $\mathrm{Mg}$ & 1.67 & 2.03 & 2.08 & 2.03 & 2.13 & 2.00 \\
\hline $\mathrm{Cl}$ & 4.02 & 4.63 & 4.21 & 4.41 & 5.1 & 4.47 \\
\hline $\mathrm{K}$ & 0.14 & - & - & - & - & 0.03 \\
\hline $\mathrm{Ca}$ & 1.01 & 1.27 & 1.19 & 1.37 & 1.65 & 1.3 \\
\hline $\mathrm{After}$
\end{tabular}

After calculating the average values of concentrations for each element, it was found that $\mathrm{C}$ and $\mathrm{O}$ were the most abundant in sepia melanin with concentration average concentrations of about $57 \%$ and $24 \%$ respectively. This analysis revealed that the major compositions of sepia melanin are $\mathrm{C}, \mathrm{O}, \mathrm{Na}, \mathrm{Cl}$, while the minor are $\mathrm{Mg}, \mathrm{Ca}, \mathrm{K}$, $\mathrm{S}$ and $\mathrm{N}$. These results serve as an additional support which reflects the purity of this sepia melanin there is no additional elemental peak. 


\subsection{TEM Micrographs}

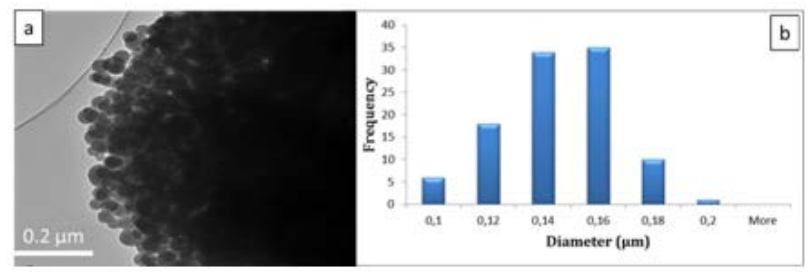

Figure 9. Typical TEM micrographs of agglomerated sepia melanin nanoparticles scaled $0.2 \mu \mathrm{m}$ and the corresponding diameter distribution histogram

TEM micrograph shows that the granules of sepia melanin are spherical (Figure 9a). The granule size was measured from TEM micrograph to determine if the diameter distribution is in agreement with that one obtained from SEM micrograph. The histogram (Figure 9b) presents the diameter distribution of small granules of sepia melanin. In decreasing the scale from $100 \mathrm{~nm}$ (Figure 10a) to $50 \mathrm{~nm}$ (Figure 10b) and increasing magnification, the structure of granule start to be a little bit clear.
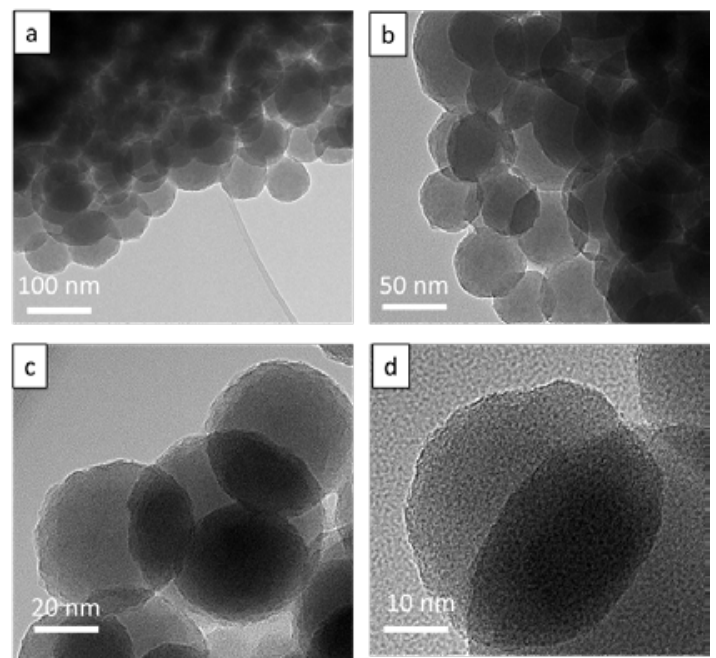

Figure 10. TEM micrographs of agglomerated sepia melanin nanoparticles scaled $100 \mathrm{~nm}$ (a), $50 \mathrm{~nm}$ (b), $20 \mathrm{~nm}$ (c), and $10 \mathrm{~nm}$ (d)

In decreasing further the scale from $20 \mathrm{~nm}$ (Figure 10c) to $10 \mathrm{~nm}$ (Figure 10d); the polymers chains forming sepia melanin start to be observed. At high magnification and 2 nm scale (Figure 11), the chains are well observed so that the distance between polymer chains can be easily measured using Image-J software.

At high magnification and $2 \mathrm{~nm}$ scale (Figure 11), the chains are well observed so that the distance between polymer chains can be easily measured using Image-J software.
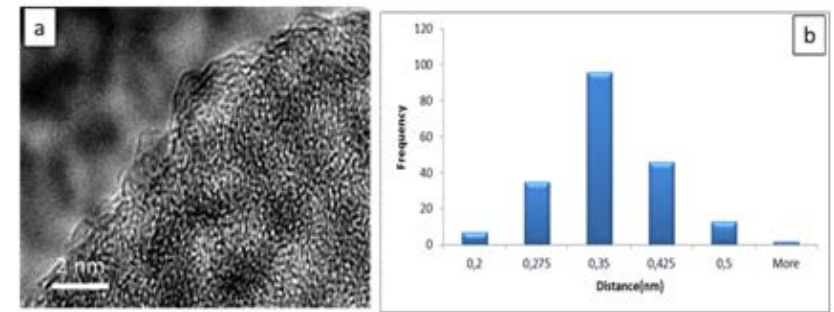

Figure 11. TEM micrograph of sepia melanin nanoparticles scaled $2 \mathrm{~nm}$ and the corresponding histogram of distances distribution between chains of polymers
The Figure 11 shows the TEM micrograph at high resolution. From TEM micrograph, it was possible to measure the distance between polymers layers of sepia melanin. The Figure $11 \mathrm{~b}$ shows the distribution of distances between chains of polymers. The average interlayer distance measured from the TEM micrograph (Figure 11a) using image-J software is $0.323 \mathrm{~nm}=3.23 \mathrm{~A}$. This confirms the supramolecular organization predicted from the corresponding TEM micrograph (Figure 11d). The obtained value is in good agreement with that one obtained by Chen et al. by simulations [27].

\section{Conclusion}

We have successful characterized the morphology of sepia melanin using SEM and TEM. The obtained results revealed that sepia melanins are formed by many aggregates agglomerated together. These aggregates are formed also by small spherical granules with different size distributions. SEM and TEM observations show that the sepia melanin powder consists of spherical nano-scaled particles with a nearly double size distribution. It was found the diameter for granules were 100 - $200 \mathrm{~nm}$. The average granule diameter measured from two different SEM micrographs was $137 \mathrm{~nm}$ and $145 \mathrm{~nm}$.

Although as yet we do not have the preferred structure of the melanin as it is highly disordered as it was found from TEM results at high resolution, the field can now move forward to do more characterisation on this commercial sepia melanin many other characterization techniques for further investigation on its morphology, and to develop a model leading to accurate and prediction of melanin structure, properties as well as its function. The fact of knowing well its structure, it will open many other new applications in different fields.

\section{Acknowledgement}

We are grateful for the funding and supports from the Organization for Women in Science for the Developing World (OWSDW), SIDA (Swedish International Development Cooperation Agency) as well as The Academy of Sciences for the developing World (TWAS), National Research Foundation of South Africa (NRF), iThemba LABS, the UNESCO-UNISA Africa Chair in Nanosciences \& Nanotechnology, and the Abdus Salam ICTP via the Nanosciences African Network (NANOAFNET) as well as the African Laser Centre (ALC).

\section{References}

[1] S. A. Centeno and J. Shamir, "Surface enhanced Raman scattering (SERS) and FTIRcharacterization of the sepia melanin pigment used in works of art,” Journal of Molecular Structure, vol. 873, p. 149-159, 2008.

[2] C. D. Derby, "Cephalopod ink:Production, Chemistry, Functions and applications,” Mar. Drugs, vol. 12, pp. 2700-2730, 2014.

[3] Z. Huang, H. Lui, X. K. Chen, A. Alajlan, D. I. McLean and H. Zeng, "Raman spectroscopy of in vivo cutaneous melanin," Journal of Biomedical Optics , vol. 9(6), p. 1198-1205, 2004.

[4] A. R. Katritzzky, N. G. Akhmedov, S. N. Denisenko and O. V. Denisko, "1H NMR spectroscopic characterization of solutions of 
sepia melanin, sepia melanin free acid and human hair melanin,” Pigment Cells Res, vol. 15(2), pp. 93-97, 2002.

[5] M. Magarelli, P. Passamonti and C. Renieri, "Purification, characterization and analysis of sepia melanin from commercial Sepia ink (Sepia officinalis),” Rev CES Med Vet Zootec, vol. 5(2), pp. 18-28, 2010.

[6] D. J. Kim, K.-Y. Ju and J.-K. Lee, "The synthetic melanin nanoparticles having an excellent binding capacity," Bull. Korean Chem. Soc, Vols. 33, No. 11, pp. 3788-3792, 2012.

[7] S. Subianto, Electrochemical Synthesis of -Like Polyindolequinone, PhD. thesis,, Inorganic material research Program, the Queensland University of Technology, 2006.

[8] K. Tarangini and S. Mishra, "Production, characterization and analysis of melanin from isolated marine pseudomonas sp. using vegetable waste.” Research Journal of Engineering Sciences, vol. 2(5), pp. 40-46, 2013.

[9] G. DJ, Dermotology, An illustrated Colour Tex, 3 rd ed. Edinburgh:Churchill Livingstone, 2002.

[10] G. Perna, M. Lasalvia, C. Gallo, G. Quartucci and V. Capozzi, "Vibrational characterization of synthetic eumelanin by means of raman and surface enhanced raman scattering," The Open Surface Science Journal, vol. 5, pp. 1-8, 2013.

[11] N. A. Alarfaj, M. A. E. Abdalla and A. M. Al-Hamza, "A Sensitive electrogenerated chemiluminescence assay for determination of melanin in natural and biological samples," International Journal of Electrochemical Science, vol. 7, pp. 7888-7901, 2012

[12] S. S. Sajjan, A. O, G. B. Kulkarni, A. S. Nayak, S. B. Mashetty and T. B. Karegoudar, "Properties and functions of melanin pigment from Klebsiella sp. GSK," Korean Journal of Microbiology and Biotechnology, vol. 41(1), p. 60-69, 2013.

[13] M. Magarelli, Purification, characterization and photodegradation studies of modified sepia melanin Sepia (Sepia officinalis).Determination of Eumelanin content in fibers from Alpaca (Vicugna pacos), Doctoral Thesis., Macerata: University of Camerino, 2011.

[14] D. Peruru, R. S, N. Ahmed VH, P. S. Sandeep, S. Raju, S. Nazan and S. Begum, "Isolation of eumelanin from Sepia officinalis and investigation of its antimicrobial activity by ointment formulation,” International Journal of Pharmacy, vol. 2(2), pp. 67-72, 2012.

[15] F. Bernsmann, O. Ersen, J.-C. Voegel, E. Jan, N. A. Kotov and V. Ball, "Melanin-containing films: Growth from dopamine solutions versus layer-by-layer deposition," ChemPhysChem, vol. 11, p. 3299-3305, 2010.

[16] D. N. Peles, Application of photoemission electron microscopy to melanin and melanosom, Duke University, 2011.

[17] S. Meng and E. Kaxiras, "Theoretical models of eumelanin protomolecules and their optical properties,” Biophysical Journal, vol. 94, p. 2095-2105, 2008.

[18] C. G. Kumar, N. Sahu, G. N. Reddy, R. Prasad, N. Nagesh and K. A., "Production of melanin pigment from Pseudomonas stutzeri isolated from red seaweed Hypnea musciformis," Letters in Applied Microbiology, 2013.

[19] G. Arun, M. Angeetha, M. Eyini and P. Gunasekaran, "Effect of copper sulphate and resorcinol on the extracellular production of melanin and laccase by Schizophyllum commune Fr. and Pleurotus cystidiosus var. Formosensis,” Indian Journal of Advances in Plant Research, vol. 1(5), pp. 55-61, 2014.

[20] J. Reisz, The spectroscopic properties of Melanin, Doctoral Thesis, Brisbane: University of Queensland, 2006.

[21] R. F. Egerton, “An Introduction to TEM, SEM, and AEM," in Physical Principles, Alberta, Edmonton: University of Alberta, 2005.

[22] S. K. Fagerland, Investigation of focused ion Beam/scanning electron microscope parameters for slice and view and Energy dispersive X-ray spectroscopy of embedded brain tissue, Master's thesis, Norwegian University of Science and Technology, 2014.

[23] J. B. Kortright and A. C. Thompson, "X-ray emmission energies," in X-Ray Data Booklet, Berkeley, Center for X-ray Optics and Advanced Light Source, 2001.

[24] D. J. Kim, K.-Y. Ju and J.-K. Lee, “The synthetic melanin nanoparticles having an excellent binding capacity,” Bull. Korean Chem. Soc, vol. 33(11), pp. 3788-3792, 2012.

[25] J.-W. Lee, H.-B. Cho, T. Nakayama, T. Sekino, S.-I. Tanaka, K. Minato, T. Ueno, T. Suzuki, H. Suematsu, Y. Tokoi and K. Niihara, "Dye sensitized solar cells using purified squid ink nanoparticles coated on Ti O2 nanotubes/nanoparticles,” Journal of Ceramic Society of Japan, vol. 121(1), pp. 123-127, 2013.

[26] F. Natalio, R. Andre, S. A. Pihan, M. Humanes, R. Weverd and W. Treme, "V2O5 nanowires with an intrinsic iodination activity leading to the formation of self-assembled melanin-like biopolymers,” Journal of Materials Chemistry, vol. 21, p. 1192311929, 2011.

[27] J. P. Bothma, Exploring the structure-propertyrelationships in eumelanin, Masters of Philosophy Thesis, School of Physical Science, Queensland University, 2008.

[28] A. A. R. Watt, J. P. Bothma and P. Meredith, "The supramolecular structure of melanin,” Soft Matter, vol. 5, p. 3754-3760, 2009.

[29] D. Moses, M. Mattoni, N. Slack, J. Waite and F. Zok, "Role of melanin in mechanical properties of Glycera jaws," Acta Biomaterialia, vol. 2, p. 521-530, 2006.

[30] C.-T. Chen, V. Ball, J. J. d. A. Gracio, M. K. Singh, V. Toniazzo, D. Ruch and M. J. Buehler, "Self-Assembly of tetramers of 5,6dihydroxyindole explains the primary physical properties of eumelanin: experiment, simulation, and design," American Chemical Society, vol. 7(2), p. 1524-1532, 2013.

[31] K. Glass, S. Ito, P. R. Wilby, T. Sota, A. Nakamura, R. Bowerse, J. Vinther, S. Dutta, R. Summons, D. E. G. Briggs, K. Wakamatsu and J. D. Simon, "Direct chemical evidence for eumelanin pigment from the Jurassic period,” PNAS Early Edition, pp. 1-6, 2012. 